\title{
LA PREGUNTA EN LAS TUTORÍAS DE LOS CENTROS DE ESCRITURA
}

\author{
QUESTIONING IN WRITING CENTER TUTORIALS
}

LA QUESTION DANS LES TUTORIELS DES CENTRES D'ÉCRITURE

\author{
Lina Calle-Arango \\ Profesional en Estudios Literarios, \\ Pontificia Universidad Javeriana, \\ Bogotá. Maestra en Edición, Uni- \\ versidad de Salamanca, Madrid. \\ Maestría en Formación de Profesores \\ de Español como Lengua Extranjera, \\ Universidad Internacional Iberoamer- \\ icana (UNINI), Puerto Rico. Estudiante \\ de Doctorado en Educación, Ponti- \\ ficia Universidad Católica de Chile, \\ Santiago, Chile. \\ Avenida Vicuña Mackenna 4860, \\ Macul, Santiago, Chile \\ linacallearango@gmail.com \\ http: //orcid. \\ org/0000-0003-4362-3075
}

\begin{abstract}
RESUMEN
Gran parte de los estudios en torno a las tutorías en los centros de escritura ha puesto en evidencia que la relación que se establezca entre el tutor y el tutorado resulta determinante en cuanto a si se cumple o no el verdadero objetivo de los centros de escritura, que desde avanzada la segunda mitad del siglo xx se determinó como la necesidad de trabajar sobre las habilidades comunicativas del individuo, y ya no sobre el producto mismo. Sin embargo, dentro de esta discusión poco espacio se le ha otorgado explícitamente al potencial del uso de la pregunta en las tutorías. En virtud de lo anterior, el presente texto teórico destaca la importancia tanto de la utilización de la pregunta como herramienta clave para fomentar el análisis y la reflexión, y cómo abordarla de manera expresa en la preparación de los tutores, específicamente aquellos que participan de los centros de escritura.
\end{abstract}

Palabras clave: centro de escritura; escritura académica; tutor par; aprendizaje colaborativo; competencia comunicativa; pregunta.

\section{Abstract}

Much of the research on tutoring in writing centers has shown that the relationship established between tutor and tutee is decisive as to whether or not the true goal of writing centers is met. That since the late second half of the twentieth century was determined as the need to work on the communicative skills of the individual, and no longer on the product itself. However, within this discussion, little space has been explicitly granted to the potential use of the question in the tutorials. In view of the above, the present theoretical text highlights the importance of using the question as a key tool to encourage analysis and reflection, and highlights the importance of addressing it explicitly in the preparation of tutors, specifically those who participate of the writing centers.

Keywords: writing center; academic writing; peer tutor; collaborative learning; communicative competence; question. 


\section{RÉSUMÉ}

La plupart des études sur les tutoriels dans les centres d'écriture a montré que la relation établie entre le tuteur et l'étudiant-tuteuré est déterminante pour savoir si le véritable objectif de ces centres est atteint ou non. En effet, depuis le début de la deuxième moitié du $\mathrm{Xx}^{\mathrm{e}}$ siècle, travailler sur les compétences de communication de l'individu, et non sur le produit est devenu une priorité. Cependant, dans cette discussion, nous soulignons le peu d'espace qui a été donné à l'importance de la question comme un outil-clé pour améliorer l'analyse et la réflexion. Ce pour ça que cette article théorique souligne l'importance d'utiliser des questions comme un outil essentiel pour encourager l'analyse et la réflexion; de plus, il souligne l'importance d'utiliser explicitement des questions dans la formation des tuteurs, spécialement ceux qui participent dans des centres d'écriture.

Mots-clés : centre d'écriture ; écriture académique ; tuteur pair ; apprentissage collaboratif ; compétence communicative ; question. 


\section{Introducción}

El nacimiento de los centros de escritura, originalmente como método y no como espacio físico, data de las primeras décadas del siglo Xx y se ubica en Estados Unidos, a partir de lo que se conocía como el método del laboratorio (Carino, 1995). Los cambios que desde entonces tuvieron lugar y que durante la segunda mitad de siglo llevaron a que se constituyera lo que hoy conocemos como "centro de escritura", pueden rastrearse en autores como Elizabeth Boquet (1999), Peter Carino (1995), Stephen North (1984; 2008), Judith Summerfield (2008), Susan Waller (2002), entre otros. Iniciando el siglo XXI, los países iberoamericanos adoptan este modelo, que poco a poco se va popularizando, afirmación que puede corroborarse en estudios como los presentados por Paula Carlino (2013), Monserrat Castelló (2014), Violeta Molina (2015; 2016) y Juan Núñez (2013). No de forma distinta sucedió en otras partes del mundo, como Asia (Johnston, Cornwell y Yoshida, 2010; Kunde, Sequeira y Patil, 2015; Tzu-Shan, 2013), Europa (Voigt y Girgensohn, 2015) y África (Van Rensburg, 2006), continentes donde actualmente pueden también encontrarse iniciativas de alfabetización académica de esta naturaleza.

Una de las motivaciones que dio origen a esta noción de "espacios de apoyo a la escritura" redundó en la necesidad de desarrollar alternativas que se alejaran de las relaciones jerárquicas tradicionales abordadas en el salón de clases. Por ello, el trabajo de pares, bajo la figura de tutorías, ha acompañado desde sus inicios esta iniciativa y continúa siendo una de las dinámicas más utilizadas en estos espacios (Calle-Arango, Pico y Murillo, 2017) alrededor del mundo. Desde la cuna de estos lugares, autores como Jeff Brooks (1991), Irene Clark (1988), Muriel Harris (2000), Susan Hubbuch (1988), Andrea Lunsford (1991) y Linda Shamoon y Deborah Burns (1995), entre muchos otros, han planteado durante décadas cuestiones relativas a los aciertos, los dilemas y las nociones erróneas en torno a las tutorías en los centros de escritura. Lo anterior, en principio, apoyados en los beneficios y las ventajas que Bruffee (1984) formuló acerca del trabajo colaborativo.

Muchos de estos estudios han puesto en evidencia que la relación que se establezca entre el tutor y el tutorado, y a partir de ello, la forma que cobre el concepto de ayuda, resulta determinante al momento de valorar si se cumple o no el verdadero objetivo de los centros de escritura. Desde avanzada la segunda mitad del siglo xx, este objetivo ha redundado en trabajar sobre las habilidades comunicativas del individuo, y ya no sobre el producto mismo. Para lograrlo, se ha puesto en evidencia la importancia de que se establezcan diálogos entre los actores de la tutoría; es decir, comprender que la conversación constituye un elemento clave para motivar los procesos cognitivos del estudiante, lo que requiere una preparación adecuada por parte del tutor. Empero, dentro de esta discusión poco espacio se le ha otorgado explícitamente al valor de la utilización de la pregunta como factor fundamental en el potenciamiento del análisis y la reflexión en asuntos relativos al desarrollo de la competencia comunicativa, quizá porque se ha obviado, o porque su correcta utilización se ha dado por sentada.

De cara a lo anterior, este artículo plantea una reflexión alrededor de la importancia de la pregunta como elemento determinante en la preparación de los tutores, específicamente de aquellos que participan de los centros de escritura. Para ello, se hace una breve contextualización inicial que sitúa al lector en lo que en la actualidad se conoce como "centro de escritura", con los paradigmas y movimientos que lo han direccionado hacia ese lugar. Posteriormente, se enfatiza en los dilemas que presenta el establecimiento -o no- de relaciones jerárquicas entre tutor y tutorado, $\mathrm{y}$ a partir de allí se pone en evidencia el uso de la pregunta como herramienta útil — de ser bien utilizadapara dirigir conversaciones que colaboren a una reflexión crítica sobre el lenguaje y ayuden a desarrollar competencias comunicativas. Por último, 
se señala cómo este proceso puede conducir a un trabajo verdaderamente colaborativo.

\section{De forma a función}

What peer tutor and tutee do together is not write or edit, or least of all proofread. What they do together is converse

Bruffee (1984, p. 2)

La proliferación, en Estados Unidos, de los centros de escritura tuvo lugar a finales de la primera mitad del siglo xx e inicios de la segunda, en su mayoría gracias a hechos coyunturales, como la integración al sistema educativo de excombatientes de la Segunda Guerra Mundial (Boquet, 1999) y, posteriormente, las políticas de admisión abierta (Boquet, 1999; Carino, 1996; Summerfield, 2008), movimiento que permitió una democratización del acceso a la educación superior. Sin embargo, debido a la naturaleza de estos sucesos, que implicaban la necesidad de preparar a aquellos que se consideraban "poco preparados" (underprepared) para afrontar los retos académicos que representaba la educación superior, el énfasis de estos lugares continuó siendo remedial. No en vano tomó fuerza la denominación "clínicas" de escritura, término que aludía, como señala Moore (1950), a espacios que se ocupaban principalmente del diagnóstico de las dificultades de escritura de cada estudiante, junto con sugerencias de medidas correctivas que podrían ser rentables. No obstante, aun cuando en muchos de tales lugares se continuaba asumiendo el nombre de "laboratorio", cuyo método homónimo fue la semilla que dio origen a la idea de estos espacios en las primeras décadas del siglo Xx (Carino, 1995; Moore, 1950; Waller, 2002).

Sin embargo, se trató también de una época en la que estudios como los de la gramática generativa de Chomsky (1974), las ideas desarrolladas por Bruner y figuras destacadas en el constructivismo, como Vygotsky, ya estaban tomando fuerza, de manera que las nociones tradicionales sobre el lenguaje estaban teniendo una sacudida significativa (Calle-Arango, 2018). En este sentido, pese a que en tales espacios de apoyo a la escritura aún se buscaba generar oraciones gramaticalmente correctas y solventar dificultades "técnicas", como las fónicas y ortográficas, los profesionales adjuntos habían emprendido una búsqueda por ampliar dicho panorama, que redundaría en un arraigo por elementos como la valoración de la composición y de la escritura como proceso, apoyados esencialmente en dinámicas de aprendizaje colaborativo y edición por pares (Summerfield, 2008).

Así, tal cambio en la noción del lenguaje hacia los años setenta permea estos espacios de apoyo en la escritura y les infunde esa idea de que se no se trata de escribir de forma "correcta", pero "vacía" de significado; sino de comunicar, como lo señalan enfoques como el comunicativo (Hernández, 1999-2000) y el concepto de competencia comunicativa planteado por Hymes (1972). Con ello comienza a parecer obsoleto pensar que estos espacios se dedicaban de manera exclusiva a subsanar asuntos puntuales de la lengua, que no redundaban realmente en la comunicación; y, de hecho, empieza a ser altamente criticada la relación entre esta idea de "tienda de reparaciones" (fix-shop) y centro de escritura:

\footnotetext{
Mientras que en el "viejo" centro la instrucción tiende a tener lugar después o aparte de la escritura, y tiende a enfocarse en la corrección de problemas textuales, en el "nuevo" centro la enseñanza se lleva a cabo, tanto como sea posible, durante la escritura, durante la actividad que se está aprendiendo, y tiende a enfocarse en la actividad misma (North, 1984, p. 439; traducción propia). ${ }^{1}$
}

No queda duda de que son este tipo de planteamientos los que llevan a dejar de considerar que tales espacios estaban destinados únicamente a los estudiantes "atrasados", y se busque abrir las puertas a toda la comunidad educativa (Kinkead, 2008).

1 En el original: "Whereas in the 'old' center instruction tends to take place after or apart from writing, and tends to focus on the correction of textual problems, in the 'new' center the teaching takes place as much as possible during writing, during the activity being learned, and tends to focus on the activity itself". 
Como curso natural de ello, hacia la década de los ochenta se evoluciona a una denominación de "centro" de escritura (Boquet, 1999), como lugar abierto en el que se reúnen las personas, usualmente uno-a-uno, para hablar sobre la escritura (Leahy, 1990), y no solo para solucionar vacíos lingüísticos. De forma consistente con el nuevo énfasis, figuras de relevancia para los centros de escritura, como North, insistieron en la necesidad de comprender que se trataba de trabajar con escritores, no con escritos; y que, por lo tanto, el texto en sí mismo no constituía el objetivo, sino el medio a través del cual se ayudaba a desarrollar tal habilidad (North, 1984). En la misma línea, en 1991, Brooks escribió: "nos sentamos con textos imperfectos, pero nuestro trabajo es mejorar a sus autores" (1991, p. 2; traducción propia). ${ }^{2}$ La atención, entonces, se volcó sobre el estudiante, quien tenía la posibilidad de mejorar sus habilidades, pese a no generar textos gramatical o sintácticamente perfectos.

A partir de esta intención por enfocarse en el desempeño del estudiante y no en sus resultados escritos, el concepto de individualidad asume otra perspectiva, pues se comienza a reconocer la pluralidad en la forma de escribir, leer y acceder al conocimiento (Summerfield, 2008). La aceptación de que los procesos de producción comunicativa varían según las características individuales del escritor constituyó el punto de ruptura entre la noción de "enseñanza del lenguaje" y las premisas base de otras disciplinas como el álgebra, que demandan respuestas únicas y verdades absolutas.

Esta noción, que fue adoptada por los centros de escritura, y que favorecía un enfoque en el proceso y el trabajo colaborativo centrado en el estudiante (Clark y Healy, 1996), encajaba a la perfección con el manejo uno-a-uno de las tutorías entre pares, dinámica que había sido constante desde los inicios de estos espacios (Calle-Arango, Pico y Murillo, 2017), y que continúa vigente como

2 En el original: "we sit down with imperfect papers, but our job is to improve their writers". uno de los elementos más comunes y de mayor éxito dentro de lo que hasta hoy se conoce como "centro de escritura" tanto en Estados Unidos (Driscoll, 2015; Hughes, Gillespie y Kail, 2010; Lunsford y Ede, 2011), en Iberoamérica (Molina, 2014; Núñez, 2013), como en otros lugares del mundo (Kunde et al., 2015; Tzu-Shan, 2013; Van Rensburg, 2006; Voigt y Girgensohn, 2015).

Si bien una de las motivaciones que llevó al uso de tutores pares fue la idea de generar espacios distintos al aula y difuminar las relaciones jerárquicas tradicionales que les son propias (Boquet, 1999; Bruffee, 1984), dicha dinámica de aprendizaje colaborativo, cuyos inicios fueron más prácticos que teóricos (Bruffee, 1984), asumió otro matiz durante la segunda década del siglo Xx, en gran parte a la luz del auge del socioconstructivismo. Cuando se comienza a valorar el proceso de escritura como herramienta que ayuda a mejorar las habilidades de producción del estudiante, la interacción entre tutor y tutorado se establece ya no a partir de una intención por "arreglar algo" o "llenar algún vacío", sino desde una dinámica de construcción conjunta de conocimientos que beneficia este proceso cognitivo.

A finales del siglo pasado, Cooper (1994) se preguntaba sobre la función que han de tener los centros de escritura, y proseguía a señalar que el papel del tutor consiste en crear conocimientos útiles sobre la escritura en la universidad y capacitar a los estudiantes como escritores que también entienden lo que implica la escritura y que actúan como agentes en ella. El objetivo de generar este aprendizaje significativo en el tutorado se alcanza en la medida en que el tutor tenga la intención genuina de indagar sobre los intereses y pensamientos del estudiante, e involucre procesos de reflexión y análisis que se distancien de la mera acción de editar un texto o señalar dónde hay errores ortográficos, sintácticos, etc. Como lo explica Brooks,

Un estudiante que acude al centro de escritura y pasivamente recibe conocimiento de un tutor no estará más cerca de su propio texto de lo que estaba cuando 
entró. Puede irse con un texto mejorado, pero no habrá aprendido mucho (1991, p. 2; traducción propia). ${ }^{3}$

Este tipo de retos desvirtúa la mecánica unidireccional, muy común en el aula de clase, sobre todo en disciplinas exactas, en que la logística se limita a transferir un conocimiento y luego a corroborar que este ha sido claramente comprendido. Fomentar ese aprendizaje por descubrimiento y mediante la reflexión demanda, muy por el contrario, la existencia de un diálogo en el que ambos actores tengan la libertad de expresar sus ideas, cuestionar e indagar sobre nuevos conocimientos. Esto resulta particularmente importante en asuntos relacionados con la escritura, si lo que se pretende es guiar al estudiante hacia niveles de alfabetización que superen los estadios de simple reproducción o incluso comunicación, para llegar a niveles como el epistémico. El potencial epistémico de la escritura "implica una forma de pensar, de crear y de evaluar críticamente el conocimiento, por lo que su realización durante los estudios universitarios constituye un desiderátum a alcanzar" (Marinkovich y Poblete, 2014, pp. 271-272).

De manera afín, Bruffee (1984) explica que, puesto que el pensamiento es una conversación interiorizada, la escritura se constituye como una conversación internalizada reexternalizada. Por lo tanto, resulta consistente plantear que, mediante la conversación (esta vez entre dos sujetos distintos, y no pensamiento-sujeto o sujeto-texto), se potencia la habilidad para expresarse mejor por escrito. No de modo distinto señala Pemberton (1995) que una de las contribuciones que los tutores han de hacer a los centros de escritura es apoyar y enriquecer el proceso cognitivo de los estudiantes, ofreciéndoles nuevas perspectivas para pensar en sus tareas, y que estas puedan posteriormente ser plasmadas de claramente sobre el papel.

3 En el original: "A student who comes to the writing center and passively receives knowledge from a tutor will not be any closer to his own paper than he was when he walked in. He may leave with an improved paper, but he will not have learned much".
Enmarcados en ese panorama, queda en evidencia la importancia de la conversación como elemento primordial de las tutorías, y el centro de escritura parece ser el espacio más adecuado para abordar estas interacciones, menos comunes en espacios tradicionales como las aulas de clase:

\section{A diferencia de los cursos tradicionales de escritura de la universidad, el enfoque de los centros de escritura no es la corrección de los problemas textuales; las tu- torías de escritura se fundamentan en las habilidades intelectuales y personales que el escritor pone en juego cuando escribe (Molina, 2014, p. 23).}

Los tutores, precisamente porque funcionan en un ambiente de apoyo no evaluativo, ofrecen a los escritores la oportunidad de escribir, pensar y hablar. Se les ayuda a usar el lenguaje para desarrollar ideas, probar posibilidades, analizar y repensar a la luz de la retroalimentación recibida (Harris, 2000). En otras palabras, el diálogo entre pares representa un contexto distinto de aprendizaje para el estudiante (Bruffee, 1984), que conduce a un trabajo colaborativo que involucra al alumno y mejora sus habilidades mediante la combinación de ejercicios como la lectura, la conversación, la escritura, y el pensamiento crítico y analítico (Lunsford, 1991), que es lo que los centros de escritura buscan potenciar.

\section{Un dilema de autoridad}

No por los señalados beneficios que trae consigo el trabajo colaborativo, y la importancia que tiene la labor que realizan los tutores pares en los centros de escritura, se pasa por alto la discusión sobre, valga la redundancia, la ausencia de paridad que se establece entre el tutor y el tutorado a la luz de estos espacios (Calle-Arango, Pico y Murillo, 2017). De manera radical, Lunsford (1991) afirmó que esa rígida jerarquía de las aulas, en la que el docente es el foco, se replica en el centro de escritura, donde el trono de autoridad es ocupado por el tutor, quien simplemente "simula" una simetría con su tutorado. En respuesta a planteamientos semejantes, Pemberton (1995) explica que, pese a que esa representación del centro de 
escritura como un lugar en el que los estudiantes y los tutores están situados en posiciones igualitarias es sin duda ingenua, esta relación es bastante diferente de la que probablemente se erige entre estudiantes y docentes, pues raramente se puede lograr ese equilibrio de poder. Quizá el elemento diferenciador radique en esa noción que Lunsford (1991) planteó como la pretensión, por parte del tutor, de no tener la misma autoridad. Y es posible que tal pretensión sea muchas veces suficiente para alivianar el peso u obstáculo que la evaluación o la nota representa para el tutorado.

En efecto, cuando se dibuja esta relación de autoridad, se hace desde la premisa de que un estudiante - el tutor - tiene mayores conocimientos o habilidades con respecto al otro - el tutorado- No obstante, en el centro de escritura se sigue gozando de la ausencia de una presión por la nota o la evaluación, cuyo peso en el aula tradicional es significativo en el desempeño de varios estudiantes, $\mathrm{y}$ en lo que reposa para muchos la relación tradicional de jerarquización:

Igualmente importante para el desarrollo de la independencia de los estudiantes como escritores es que puedan plantear preguntas más honestas a los tutores en el entorno colaborativo de una tutoría. Con demasiada frecuencia, estas preguntas son las que los profesores desearían que los alumnos hicieran en clase, pero son las que no hacen, debido a un temor equivocado de parecer inadecuados (Harris, 2000, p. 110; traducción propia). ${ }^{4}$

A ello se suma el hecho de que el conocimiento del tutor, por ser la mayoría de las veces un estudiante no graduado, no sobrepasa significativamente el de su tutorado (Roscoe y Chi, 2007). Esta ausencia de un conocimiento notoriamente avanzado sin duda disminuye la brecha de autoridad

4 En el original: "Equally important to students' developing independence as writers is that they can ask peer tutors more honest questions in the collaborative setting of a tutorial. Such questions are all too often the ones teachers wish students would ask in class but ones that they won't because of a mistaken fear of appearing inadequate".
(Hubbuch, 1988; Pemberton, 1995) y potencia los resultados significativos de la conversación:

[...] aunque ni los tutores ni sus tutorados pueden dominar por sí solos el discurso normal de una comunidad de conocimiento dada, trabajando en conjunto - compartiendo sus recursos - es muy probable que puedan dominarlo si su conversación está estructurada indirectamente por la tarea o problema que un miembrodeesacomunidad(eldocente) propone(Bruffee, 1984, p. 11; traducción propia). ${ }^{5}$

De ahí que no es la relación en sí la que provee el carácter de verticalidad u horizontalidad entre los actores, sino la forma como esta es abordada por ellos. Por esta razón, se insiste en la importancia de conversaciones que ejerciten la habilidad reflexiva y analítica, y verdaderamente aporten para hacer del estudiante un mejor escritor, pero también un individuo más crítico.

En rigor de lo expuesto, Ashton-Jones (1988) señaló como uno de los inconvenientes de las interacciones entre tutor y tutorado la ambigüedad producida respecto del verdadero papel del tutor, y el conflicto de valores generado en este, quien lucha por afirmar la lealtad tanto a sus compañeros como a un sistema académico que ha reconocido sus capacidades. De ello surgen dilemas, como no saber si debe tomar el lápiz y hacer correcciones sobre el texto (como un "pequeño" docente), o bien incitar libremente a la reflexión de su tutorado (como un "compañero"), a veces con el miedo de evidenciar vacíos que, "se supone", no debería tener —en su calidad de tutor-. A partir de ello, quizá el tutor prefiera limitarse a establecer preguntas cuya respuesta conoce, con el objetivo de mantenerse en un "terreno seguro". Tal presión es la que en forma eventual constituye un reto para la manera como se procede en

5 En el original: "while neither peer tutors nor their tutees may alone be masters of the normal discourse of a given knowledge community, by working together-pooling their resources-they are very likely to be able to master it if their conversation is structured indirectly by the task or problem that a member of that community (the teacher) provides". 
las sesiones de tutorías. A veces, incluso, el mismo estudiante espera que el tutor no sea par, sino que de modo directo le ayude a arreglar su texto. Esta visión de que los tutores están ahí para "arreglar" ha sido ampliamente señalada como errónea por autores de distintas procedencias (Brooks, 1991; Clark, 1988; Clark y Healy, 1996; Kunde et al., 2015; Tan, 2011; Tzu-Shan, 2013).

En concordancia con lo anterior, la preparación del tutor no debe enfatizar en el perfeccionamiento de sus habilidades de lectura y escritura con miras a apoyar el proceso de sus pares, sino en ejercitarse en la forma acertada de abordar una sesión de tutoría, de manera en que esta sea significativa para el tutorado en lo que respecta a sus capacidades de escritura y procesos cognitivos involucrados. Con ello, por demás, se aliviana esa mencionada presión que sienten algunos tutores en relación con tener la verdad absoluta o evidenciar que saben. Estos podrán realmente centrarse en aportar a su tutorado en un proceso ya no de transmitir conocimiento, sino de apoyar su descubrimiento. No en vano, hacia mediados de los ochenta, comenzaron a aparecer varios artículos en el Writing Center Journal y en el Writing Lab Newsletter en los cuales se motivaba a los centros de escritura a reclutar tutores de todas las disciplinas (Kiedaisch y Dinitz, 2008), precisamente con el objetivo de prevenir, o al menos disminuir, la reproducción de esas relaciones jerárquicas tradicionales. Cuando el foco está puesto en la competencia comunicativa y el potencial epistémico de la escritura, resulta más beneficioso que el tutor sea ajeno al lenguaje de cierta disciplina, en tanto que esto forzará al estudiante a simplificar, reordenar, desagregar y evaluar sus ideas lo suficiente como para lograr una transferencia exitosa a distintos tipos de receptores. En otras palabras, dará lugar a procesos cognitivos que contribuyan a lograr mayores habilidades de comunicación.

En esa línea, la falta de familiaridad del tutor con las convenciones del discurso que atañe a la tarea se convierte en una de sus mayores fortalezas (Pemberton, 1995), toda vez que le permitirá adherirse más naturalmente a una conversación que propenda por el descubrimiento y la construcción de saberes. Este tipo de tutor ha sido llamado "tutor genérico" (generalist tutor), y se distingue de aquel que es conocedor de la disciplina en la que su tutorado está inscrito. El "tutor conocedor", por su parte, se encuentra más propenso a caer en la tentación de "apropiarse" del texto del estudiante (Hubbuch, 1988; Kiedaisch y Dinitz, 2008), y muchas veces tiende a poner énfasis en el producto, en lograr formas "correctas", en vez de concentrarse en el desarrollo del escritor. En suma, el conocimiento del tutor respecto de cierta disciplina no resulta de ninguna manera garantía para que la tutoría sea exitosa; puede, incluso, resultar peligroso si el tutor no sabe establecer estos límites. Por esto, no sorprende que muchos docentes que no pertenecen a los centros de escritura hayan cuestionado ampliamente la labor de tales espacios, con el argumento de que, al enviar a sus estudiantes a mejorar sus procesos, se exponen a que la tarea no termine siendo realizada por ellos (Clark, 1988; Clark y Healy, 1996).

Así mismo, como bien señalan Kiedaisch y Dinitz (2008), más de la mitad de los productos que los estudiantes buscan llevar a cabo a lo largo de su formación académica superior están dirigidos a un público general, de manera que requieren no tanto un apoyo de alguien experto en la disciplina, sino más bien alguien que pueda ayudarles a desarrollar sus estrategias comunicativas y reflexionar sobre sus decisiones de escritura. Es esta la razón por la cual resulta determinante que el tutor sea consciente de que su misión se centra en potenciar el pensamiento crítico de su tutorado, guiarle en un descubrimiento que lo conduzca a aclarar sus dudas y superar sus dificultades comunicativas; y, sobre todo, facilitarle el proceso, sin imponer sus ideas o su propio conocimiento (Shamoon y Burns, 1995). No obstante, esta conciencia puede no ser suficiente si ellos no saben cómo ponerla en práctica, puesto que, aun cuando los tutores 
intentan establecer relaciones basadas en igualdad entre pares, en muchas ocasiones es probable que reproduzcan, como se mencionó, la estructura jerárquica en la que el profesor transmite el conocimiento a los estudiantes (Ashton-Jones, 1988), dado que corren el riesgo de pensar que "saben hacer mejor la tarea", y quizá porque tal modelo es el único ejemplo pedagógico que conocen.

Justamente, el uso de la pregunta es una de las herramientas más útiles para evitar cruzar esa línea fina entre incitar a la reflexión crítica y corregir la tarea del estudiante, además de que formular preguntas realmente indagatorias, propias de un método no dirigido, ayuda a conservar la relación de paridad (Carino, 2003). Pero incluso algo que podría parecer tan sencillo, como el ejercicio de cuestionar, tiene sus aristas; por esta razón, es necesario preparar sobre la manera en que se debe abordar tal quehacer.

Una investigación efectuada por Roscoe y Chi (2007) en relación con el comportamiento de los tutores en diferentes edades y niveles académicos puso en evidencia que la mayoría de las preguntas que estos enunciaban se centraba en hechos básicos. Adicionalmente, era frecuente el uso de preguntas cerradas, de verificación, es decir, el planteamiento de un interrogante para solicitar confirmación de una afirmación que se conoce o se considera correcta (Roscoe y Chi, 2007), lo que no solo limita las posibilidades analíticas del tutorado, sino que mantiene al tutor en una zona de confort del conocimiento, sobre todo cuando se habla de ejercitar procesos cognitivos y desarrollar la competencia comunicativa.

\section{La pregunta como indagación}

Dentro de la literatura más representativa que da cuenta de los centros de escritura desde sus diversas características se halla poca mención explícita a la formulación de preguntas por parte de los tutores como una de las dinámicas más recurrentes en estos espacios. Sin embargo, se ha reconocido, ya no en los centros de escritura, sino a nivel general, que la pregunta es uno de los recursos más difundidos y fundamentales de la tutoría (Roscoe y Chi, 2007; 2008). Esto sucede debido a la pluralidad de formas y posibilidades que la pregunta puede representar, y, en este caso, porque es susceptible de adaptarse al paradigma comunicativo de cualquier época. Lo anterior en tanto que, como afirma Zuleta Araújo (2005), la conversación interpersonal se encuentra íntimamente ligada a la dinámica de pregunta y respuesta, por lo que está siempre presente como recurso pedagógico y, por ende, como oportunidad para abrirse al conocimiento.

Boquet (1999) menciona el auge del enfoque psicoterapéutico de la década de los cuarenta que, encabezado por Davidson y Sorenson, ofrecía a los educadores otro modo de pensar las formas de regular el comportamiento. Este enfoque, señala la autora, contribuyó a legitimar los centros de escritura con una aproximación más cercana al cognitivismo que al estructuralismo (Boquet, 1999, p.469). En esta línea, la Terapia no directiva de Carl Rogers (Rogerian non-directive counseling) se centraba en una dinámica de preguntas que dirigían al autodescubrimiento, y los centros de escritura proporcionaban un espacio donde los estudiantes podían sentirse seguros en su expresión de pensamientos e ideas (Boquet, 1999). A partir de ello, en la literatura sobre tutores y centros de escritura es usual encontrar alusiones a las tutorías no directivas (non-directive tutoring) (Carino, 2003; Clark y Healy, 1996; Shamoon y Burns, 1995), que linda con la premisa del constructivismo social respecto de la adquisición del lenguaje mediante los procesos de interacción social del individuo (Vygotsky, 2001) y, por tanto, la escritura como un acto colectivo, más que un proceso de descubrimiento personal y de expresión individual. Así mismo, Brooks (1991), al hacer referencia a la Tutoría Minimalista Avanzada (Advanced Minimalist Tutoring), reconoce la importancia de que el estudiante hable, y para ello el tutor debe generar preguntas que lo 
inciten a expresarse. Una idea semejante es señalada por Waldo, quien afirma que "El programa de tutorías del centro de escritura complementará la consultoría, desarrollando preguntas apropiadas y estrategias de colaboración para inducir a los estudiantes a que hagan mejoras en sus documentos" (1993, p. 23; traducción propia). ${ }^{6}$

Llama también la atención el artículo de AshtonJones, "Asking the right questions: A heuristic for tutors" (1988), en el que se habla del método socrático como esencial para la construcción de las tutorías. La autora presenta la tutoría heuristica como una herramienta mediante la cual los tutores generan el tipo de preguntas que podrán realizar a los tutorados. A ella la referencian posteriormente autores como Clark y Healy (1996) y Shamoon y Burns (1995), en sus artículos respecto del uso de tutorías. Sin duda, esta alusión a la mayéutica o dialéctica es clave. Incluso cuando North postula los centros de escritura como lugares que deben ser aceptados como espacios cuya principal responsabilidad y única razón de ser es hablar con los escritores, alude a Sócrates como un tutor ateniense que "estableció el mismo tipo de negocio: abierto a todos los interesados, sin cobro de honorarios, para ofrecer, en cualquier tema que un visitante propusiera, una dialéctica continua que es, a fin de cuentas, su razón de ser" (North, 1984, p. 446; traducción propia). ${ }^{7}$

No obstante, las investigaciones sobre el cuestionamiento en las aulas han demostrado que los estudiantes son reticentes a la enunciación de preguntas, sobre todo de aquellas que demandan respuestas significativas. Este comportamiento ha sido generado en gran parte por la dificultad de identificar o expresar sus confusiones, o bien

6 En el original: “The writing center's tutoring program will complement the consultancy by developing appropriate questions and collaborative strategies for drawing students to make improvements on their papers".

7 En el original: "set up the same kind of shop: open to all comers, no fees charged, offering, on whatever subject a visitor might propose, a continuous dialectic that is, finally, its own end". por temor a evidenciar ignorancia (Roscoe y Chi, 2007). Dada esta situación, y teniendo en cuenta que los tutores son también estudiantes, sería un error dar por sentado que estos no temen o no son resistentes a hacer preguntas a sus tutorados, o al menos aquellas que conduzcan a la reflexión y al análisis significativo, sobre todo si se le suma el mencionado papel "jerárquico" en la relación. Aunado a ello, téngase presente la ya señalada complejidad de que estas interacciones, en especial en los centros de escritura, deban plantear cuestionamientos epistemológicos y de reflexión, que pocas veces constituyen verdades absolutas o respuestas correctas, y que redundan en la argumentación y en las opiniones personales. En contraste, los tutores de disciplinas como las matemáticas pueden libremente limitar sus preguntas a respuesta cerrada o corroboración, puesto que el énfasis recae sobre la comprensión de una verdad ya establecida a partir de un proceso lógico invariable.

De acuerdo con lo dicho, basándose en las dificultades de definir el papel y las responsabilidades de los tutores pares en los centros de escritura, y a partir de lo planteado por Bruffee (1984) en cuanto a la importancia de la conversación como parte fundamental del proceso de aprendizaje, es primordial formar al tutor en cómo puede, a través de un proceso de autoinvestigación, generar un diálogo socrático, que aborde preguntas que ayuden a sus tutorados a reflexionar sobre sus decisiones de escritura (Ashton-Jones, 1988). Aunque la aproximación de la tutoría heurística da luces sobre cómo proseguir en la formación de tutores que hagan las preguntas más acertadas, la propuesta presentada por Ashton-Jones (1988) resulta limitada: sus cuatro énfasis -Estableciendo empatía (Establishing Rapport), explorando el potencial (Exploring Potential), descubriendo estrategias (Discovering Strategies) y autorrevisándose continuamente (On-Going SelfReview) - dan cuenta de tipos de preguntas que el tutor podría hacerse a sí mismo para mejorar sus estrategias y metodologías, pero no respecto al tipo de preguntas más o menos convenientes que podrán hacer a los estudiantes con 
el objetivo de estimular el crecimiento intelectual e incitar al análisis crítico.

Tal como reportan Sadker y Sadker (1993), los estudios realizados en los años setenta evidencian que, para entonces, la mayoría de los docentes continuaba utilizando la pregunta como herramienta básica para el aprendizaje, pero estas preguntas, en su mayoría, dependían por completo de repeticiones memorísticas de respuestas correctas. Este tipo de pregunta cuya respuesta es conocida por el profesor no es la que nos concierne; atañe acá la pregunta de un par, de alguien que genuinamente busca indagar, saciar una curiosidad. Zuleta Araújo lo expresa así: "Para poder preguntar hay que querer saber, esto es, saber que no se sabe" (2005, p. 117). En términos del lenguaje como disciplina, esta diferencia marca la verdadera brecha entre la jerarquía tradicional académica y la de pares en centros de escritura: la motivación con la que una pregunta se hace. Es a la luz de esa premisa que podrían evaluarse los tipos de preguntas por forma, propuestas en su momento por Widdowson (1978): 1) preguntas informativas (Wh-questions), 2) preguntas polares (Polar questions), 3) evaluaciones de verdad (Truth assessments), y 4) selección múltiple (Multiple choice). En términos generales, los dos primeros tipos son las que competen en este panorama, dado que, de ser formuladas con respuesta abierta, demandan al estudiante procesos cognitivos que permitan la capacidad de comunicarse efectivamente. Respecto a los dos últimos tipos, estas deberían ser preguntas, por ejemplo, de verificación, en las que el tutor consulta sobre lo que busca conseguir el estudiante y establece los límites a partir de los cuales se llevará a cabo la interacción - tipo de tarea asignada, criterios otorgados por el docente de la materia, avances y dudas que motivan al estudiante a solicitar apoyo, etc.- - , pero no constituyen la interacción de la tutoría en sí misma.

Sadker y Sadker (1993), por su parte, clasifican las preguntas a partir de los seis niveles (conocimiento, comprensión, aplicación, análisis, síntesis y evaluación) de la taxonomía de Bloom, puesto que en cada uno de estos se requiere un proceso cognitivo distinto. Pese a que en el aula de clase los seis niveles cobran su respectiva importancia y ninguno de estos debe ser omitidos, no sucede lo mismo en las tutorías de los centros de escritura, debido a la naturaleza de la relación apropiada entre tutor y tutorado.

Al tutor no le corresponde, por ejemplo, la formulación de preguntas de conocimiento (primer nivel), no solo porque no hay garantía de que este tenga conocimiento de la disciplina, sino porque es el estudiante el que habrá de responsabilizarse, en este punto del proceso, sobre su aprendizaje; y porque, en su mayoría, estas preguntas de corroboración no redundan en capacidades comunicativas, sino memorísticas.

Algo semejante sucede con las preguntas de comprensión (segundo nivel), que "requieren que el estudiante demuestre que posee una comprensión suficiente para organizar y ordenar mentalmente un material" (Sadker y Sadker, 1993, p. 182). Sin embargo, estas sí reclaman cierta presencia en las tutorías, pero su función es, por decirlo de alguna manera, de contextualización. Es decir, si bien con su formulación el tutor no busca comprobar — como el docente- un conocimiento, su respuesta le es útil como herramienta de apertura a una tutoría, con el objetivo de contextualizar a su emisor y delimitar el campo dentro del que tendrá lugar la sesión.

El punto de quiebre se sitúa en las preguntas de tercer nivel, puesto que son estas las que en general motivan al estudiante a asistir al centro de escritura. En este nivel de aplicación redunda la presión jerárquica estudiante-profesor, en tanto que el primero debe ser capaz de aplicar lo aprendido siguiendo las instrucciones que le han sido dadas. Dada su naturaleza, pocas veces el tutor se ocupa de estas preguntas; al contrario, ofrece su soporte para que estas sean resueltas, como se ha señalado anteriormente, mediante un apoyo al desarrollo de las habilidades del tutorado. De lo contrario, 
nuevamente, se estaría trabajando con textos, y no con escritores.

Algo por completo distinto es lo que sucede con los tres últimos niveles, constituidos por preguntas de tipo complejo, que no precisan respuesta única y que, por tanto, exigen que el estudiante ponga en marcha funciones ejecutivas que apoyen procesos de análisis y realización de inferencias. Las preguntas de análisis - cuarto nivel- convocan que el estudiante piense críticamente e infiera a partir de determinados conocimientos. Este tipo de preguntas son también denominadas "de inferencia”. Según Vail, Grafsgaard, Boyer, Wiebe y Lester (2016), estas preguntas de inferencia, que demandan del estudiante un razonamiento sobre el conocimiento del contenido o la formulación de un plan, estimulan el desequilibrio cognitivo en los estudiantes, paso crucial en la adquisición de conocimiento. Ejemplos de este tipo de preguntas son: “‘Cómo se puede corregir este error?’; ‘Cómo crees que se puede resolver este problema?"' (Vail et al., 2016, p. 157).

Las preguntas de sintesis — quinto nivel-, por su parte, solicitan la originalidad y la creatividad del estudiante para generar una respuesta, mientras que laspreguntas de evaluación - sexto nivel-dan cabida a la expresión de opiniones del estudiante, o su capacidad para evaluar desde una óptica argumentativa y sustentada (Sadker y Sadker, 1993). Estas últimas implican un carácter evidentemente distinto al del aula de clase, dada la ausencia de una nota evaluativa. En suma, estos tres últimos niveles agrupan aquellas preguntas que demandan esfuerzos cognitivos que conducirán a un aprendizaje significativo, toda vez que requieren de los estudiantes hacer uso de conocimientos previos, establecer relaciones e inferencias, y arriesgarse a la argumentación sustentada.

Las preguntas de primer y tercer nivel —y del sexto, desde la existencia de una nota- son aquellas que más marcan las jerarquías tradicionales entre el docente y el estudiante, y, por lo tanto, han de ser evitadas por el tutor, en el entendido de la tutoría como un espacio de colaboración en función de una mejor comunicación, que fomenta procesos de pensamiento y análisis, y prescinde de procesos de corroboración de conocimientos transferidos o adjudicación de criterios evaluativos sobre estos. Las preguntas complejas, por su parte, que exigen análisis más que memoria, aumentan el logro en el alumno y estimulan el pensamiento, y llevan a un mejor desempeño (Sadker y Sadker, 1993):

\section{$\mathrm{Al}$ preguntar a los estudiantes: ¿qué quieres decir con esto?", los tutores ayudan a los estudiantes a darse cuenta de que los lectores a menudo necesitan más información o explicaciones para entender lo que los escritores tenían en mente. Al preguntarles a los alumnos: “¿Cuál es tu razón para poner $Q$ antes que $\mathrm{N}$ ?", y preguntas similares, los tutores ayudan a los estudiantes a pensar en las decisiones que toman al escribir como algo razonable y no simplemente como una cuestión de seguir reglas (Cooper, 1994, p. 56; traducción propia). ${ }^{8}$}

Existen diferentes clases de preguntas quelos tutores pueden generar con el objetivo de abrir camino o incitar al análisis, construir nuevas ideas, cuestionarse sobre las planteadas e inferir. Según Roscoe y Chi (2007; 2008), algunos tipos de preguntas que los tutores hacen y que potencian estos aspectos son aquellas de revisión para introducir temas y remover conocimientos previos (“¿Este tipo de trabajo te recuerda a otro que hayas hecho?, ‘Cómo solucionaste esa situación?’, ¿Qué relación puedes establecer entre lo que estamos hablando y lo que ya conoces sobre el tema?”); preguntas a partir de las cuales valorar qué ha comprendido el estudiante, como la solicitud de parafraseo; o bien preguntas de tipo hipotético, que estimulan un razonamiento más profundo (“¿Qué diría una persona que no estuviera de acuerdo con tu posición?”).

8 En el original: "By asking students 'What do you mean by this?' tutors help students learn that readers often need more information or explanation in order to understand what writers had in mind. By asking students, 'What's your reason for putting Q before N?' and similar questions, tutors help students to think about the decisions they make in writing as reasonable rather than simply a matter of following rules". 


\section{Un aprendizaje colaborativo}

Es justo señalar, en virtud de lo mencionado, que una tutoría que se ajuste a los parámetros que se han venido exponiendo, a saber, en los que se mantenga una paridad y primen los procesos inferenciales, la construcción de conocimiento y el descubrimiento, constituirá también un aprendizaje para el tutor en sí mismo. En primer lugar, el acto de construir preguntas ha evidenciado ser una estrategia fundamental para la autorregulación cognitiva, además de fortalecer la concentración (Rosenshine, Meister y Chapman, 1996). La construcción de preguntas requiere, como señalan Galicia, Sánchez y Ventura (2105), un proceso cognitivo que implica una serie de elementos y habilidades para que el tutor, en este caso, pueda identificar el conocimiento y el nivel de abstracción de su tutorado. A ello se le suma que el tutor se verá obligado a ensayar sus conocimientos e integrar estos con otros nuevos para generar más ideas (Roscoe y Chi, 2008), negociar, argumentar y reflexionar. Dicho de otro modo, tanto para el tutor como para el tutorado resulta más beneficioso el ejercicio de enunciar preguntas de tipo complejo o inferenciales, que exijan la integración de previos y nuevos conocimientos, así como la reorganización de modelos mentales, generación de inferencias y autocontrol metacognitivo (Roscoe y Chi, 2007).

Es decir, en síntesis, que se da lugar a una interacción que implica un ejercicio fructífero y de aprendizaje significativo para el tutor mismo, y la tutoría se consolida como un espacio de negociación más que de enseñanza, en donde el ejercicio inferencial guía una búsqueda razonada por encontrar el conocimiento y la formulación conjunta de planes para la resolución de problemas (Vail et al., 2016). Ejemplo de ello es el proceso de construcción de estructuras precategoriales, o planes de escritura conjuntos, que demandan del tutorado el reto de organización lógica comunicativa; y del tutor, la habilidad de hacer las preguntas correctas que guíen al primero, sin imponer sus ideas o estrategias de composición. Con las preguntas de tipo complejo se incita al descubrimiento; a considerar distintas posibilidades en busca de una que se adecúe o que apoye argumentativamente una posición, pero también se obliga al tutor a lograr niveles de exigencia mayores, a salir de su zona de confort: en otras palabras, se logra una dinámica en la cual ambos actores están construyendo conocimiento:

Para generar preguntas de integración y razonamiento, los tutores también deben reflexionar sobre las ideas, las relaciones y los principios fundamentales necesarios para producir una respuesta correcta. Por lo tanto, la formulación de preguntas puede ayudar a los tutores a reforzar y organizar aún más su propia comprensión (Roscoe y Chi, 2007, p. 553; traducción propia). ${ }^{9}$

De este modo se da lugar, a cabalidad, a un trabajo colaborativo; un camino de doble sentido (Bruffee, 1984; Roscoe y Chi, 2008; Waller, 2002). Tutor y tutorado habrán logrado, tras la sesión, mayores niveles de comprensión y abstracción de sus ideas.

\section{Consideraciones finales}

Pese a las diferencias actuales entre los distintos centros de escritura alrededor del mundo, su objetivo coincide en una búsqueda por brindar un ambiente de aprendizaje colaborativo que se distinga del presentado en el aula de clase (Molina, 2016). Es esta la razón por la cual la figura de tutorías como estrategia para evitar las relaciones jerárquicas académicas tradicionales ha estado presente desde los inicios de los centros de escritura, incluso desde principios del siglo XIX, cuando aún se consideraba la enseñanza de la lengua desde una óptica meramente lingüística y normativa. El cambio en la concepción de la enseñanza del lenguaje, que tuvo lugar en la segunda mitad del mismo

9 En el original: “To generate integration and reasoning questions, tutors may have to also reflect upon the fundamental ideas, relationships, and principles needed to produce a correct answer. Thus, question-asking may help tutors further reinforce and organize their own understanding”. 
siglo, dio paso a una nueva forma de abordar la escritura, que a su vez condujo a que los centros de escritura levantasen su rótulo como espacio de apoyo de carácter remedial. La, para entonces, "novedosa" intención de trabajar con escritores en vez de con escritos y, por tanto, atender a los procesos y no a los productos, otorgó mayor relevancia a las tutorías entre pares, como dinámica idónea para generar espacios de construcción de conocimiento, y fomentar la reflexión y el pensamiento crítico del tutorado.

Sin embargo, muchos estudiantes están acostumbrados a que sus profesores les evalúen el producto y, por lo tanto, tienden a atribuirle más importancia a este, como si el ejercicio de esa escritura se encontrara desvinculado por completo de los anteriores o posteriores productos, obviando la necesidad de desarrollar una conciencia y una habilidad que aporte a sus otros procesos de aprendizaje. Se trata, posiblemente, de un problema de inmediatez. En este sentido, continúa siendo difícil borrar del pensamiento colectivo la idea del centro de escritura como entorno de trabajo remedial. La realidad, empero, es que el estudiante no debe acudir a este lugar a preguntar, sino a responder preguntas que le ayuden a descubrir, por sí mismo, qué es lo que desea construir con el lenguaje. El uso de la pregunta de carácter indagatorio y de tipo complejo, además de ayudar a conservar la horizontalidad entre los actores, propicia procesos de análisis y pensamiento que contribuyen a la formación de individuos más críticos y competentes, con capacidad de argumentar sus decisiones de escritura. Así, los estudiantes logran encontrar formas más adecuadas de expresar sus ideas, ejercicio que trascenderá el aprendizaje de una tarea aislada, y que implicará niveles epistémicos de alfabetización.

En atención a lo anterior, es preciso que en los centros de escritura se prepare a los tutores para ayudar a formar mejores escritores y no solo mejores textos, y que esta preparación redunde de manera explícita en el modo adecuado de plantear preguntas que conduzcan a una genuina conversación indagatoria y reflexiva. Este ejercicio constituye, a su vez, una herramienta de aprendizaje también para el tutor en cuestión, quien deberá negociar, argumentar y reflexionar, junto con su tutorado, en la dinámica propia de una conversación que construye conocimiento. Tal panorama devela una situación de aprendizaje verdaderamente colaborativo, es decir, de carácter bidireccional.

Por último, y como respuesta a lo presentado, se considera pertinente, como futuro camino de indagación, que dentro de los escenarios pedagógicos en los que las tutorías cumplen un papel protagónico, como sucede en los centros de escritura que apoyan de manera transversal a las instituciones en la formación del ciclo superior, se realicen estudios empíricos que den luces sobre estas tendencias, y analicen de manera focalizada el uso de la pregunta, su impacto y su aplicación práctica. Lo anterior, con miras a tener bases más robustas sobre los procesos que ocupan a estos lugares, y poder continuar trabajando en el mejoramiento de iniciativas que apoyen una alfabetización académica de calidad.

\section{Referencias}

Ashton-Jones, E. (1988). Asking the right questions: A heuristic for tutors. Writing Center Journal, 9(1), 29-36.

Boquet, E. (1999). "Our little secret": A history of writing centers, pre- to post-open admissions. College Composition and Communication, 50(3), 463-482.

Brooks, J. (1991). Minimalist tutoring: Making the student do all the work. Writing Lab Newsletter, 15(6), 1-4.

Bruffee, K. (1984). Peer tutoring and the "Conversation of Mankind". En G. Olson (Ed.), Writing Centers: Theory and Administration (pp. 3-15). Urbana, IL: NCTE.

Calle-Arango, L. (2018). Educación superior: la alfabetización en géneros discursivos. Educação \&o Realidade, 43(2): 629-651.

Calle-Arango, L., Pico, A. L., y Murillo, J. H. (2017). Los centros de escritura: entre nivelación académica y construcción del conocimiento. Cadernos de Pesquisa, 47(165), 872-895.

Carino, P. (1995). Early writing centers: Toward a history. The Writing Journal Center, 15(2), 103-115. 
Carino, P. (1996). Open admissions and the construction of writing center history: A tale of three models. The Writing Center Journal, 17(1), 30-48.

Carino, P. (2003). Power and authority in peer tutoring. En M. Pemberton y J. Kinkead (Eds.), The Center Will Hold. Critical Perspectives on Writing Center Scholarship (pp. 97-113). Colorado: University Press of Colorado.

Carlino, P. (2013). Alfabetización académica diez años después. Revista Mexicana de Investigación Educativa, 18(57), 355-381.

Castelló, M. (2014). Los retos actuales de la alfabetización académica: estado de la cuestión y últimas investigaciones. Enunciación, 19(2), 346-365.

Chomsky, N. (1974). Estructuras sintácticas. México: Siglo Veintiuno Editores.

Clark, I. (1988). Collaboration and ethics in writing centers pedagogy. The Writing Center Journal, 9(1), 3-12.

Clark, I., y Healy, D. (1996). Are writing centers ethical? Writing Program Administration, 20(1/2), 32-48.

Cooper, M. (1994). Really useful knowledge: A cultural studies agenda for writing centers. The Writing Center Journal, 14(2), 97-111.

Driscoll, D. (2015). Building connections and transferring knowledge: The benefits of a peer tutoring course beyond the writing center. The Writing Center Journal, 35(1), 153-181.

Galicia, I., Sánchez, H., y Ventura, P. (2015). Contenido social-experimental de las asignaturas: su influencia en las preguntas de alumnos y profesores universitarios. Revista de Investigación Educativa, 21, 121-147.

Harris, M. (2000). The writing center and tutoring in WAC Programs. In S. McLeod y M. Soven (Eds.), Writing Across the Curriculum: A Guide to Developing Programs (pp. 109-122). California: Sage Publications.

Hernández, F. L. (1999-2000). Los métodos de enseñanza de lenguas y las teorías de aprendizaje. Encuentro, 11, 141-153.

Hubbuch, S. (1988). A tutor needs to know the subject matter to help a student with a paper: ___ Agree disagree _ not sure. The Writing Center Journal, $8(2), 23-30$

Hughes, B., Gillespie, P., y Kail, H. (2010). What they take with them: Findings from the peer writing tutor alumni research project. The Writing Center Journal, 30(2), 12-46.
Hymes, D. (1972). On communicative competence. En J. B. Pride y J. Holmes (Eds.), Sociolinguistics (pp. 269293). Harmondsworth: Penguin.

Johnston, S., Cornwell, S., y Yoshida, H. (2010). Writing centers and tutoring in tutoring in Japan and Asia. JALT2009 Conference Proceedings, 692-701.

Kiedaisch, J., y Dinitz, S. (2008). Look back and say "so what": The limitations of the generalist tutor. En R. Barnett y J. Blumner, The Longman Guide to Writing Center Theory and Practice (pp. 260-271). Nueva York: Pearson Education.

Kinkead, J. (2008). The National Writing Centers Association as mooring: A personal history of the first decade. En R. Barnett y J. Blumner, The Longman Guide to Writing Center Theory and Practice (pp. 2940). Nueva York: Pearson Education.

Kunde, N., Sequeira, V., y Patil, M. (2015). A Writing Centre in India - A Case Study of the Writing Centre at Parvatibai Chowgule College of Arts and Science, Goa, India. International Journal of Educational Planning \& Administration, 5(1), 11-18.

Leahy, R. (1990). What the college writing center is-and isn't. College Teaching, 38(2), 43-48.

Lunsford, A. (1991). Collaboration, control, and the idea of a writing center. The Writing Center Journal, 12(1), 3-10.

Lunsford, A., y Ede, L. (2011). Reflections on contemporary currents in writing center work. The Writing Center Journal, 31(1), 11-24.

Marinkovich, J., y Poblete, C. (2014). Alfabetización en escritura académica en Biología: propósitos comunicativos y niveles de alfabetización. Onomázein, 30, 269-285. DOI: https://www.doi.org/10.7764/ onomazein. 30.20

Molina, V. (2014). Centros de escritura: una mirada retrospectiva para entender el presente y futuro de estos programas en el contexto latinoamericano. Legenda, $18(8), 9-33$.

Molina, V. (2016). Capítulo XIII. Los centros de escritura en Latinamérica: consideraciones para su diseño e implementación. En G. Bañales, M. Castelló y N. Vega (Coords.), Enseñar a leer y escribir en la educación superior. Propuestas educativas basadas en la investigación (pp. 339-362). México: Universidad Autónoma de Tamaulipas, fundación SM, CEIDEA, Consejo Puebla de Lectura A. C. Recuperado de http://www.fundacion-sm.org.mx/sites/default/ files/Ense\%C3\%B1ar\%20a\%20leer\%20y\%20escribir.pdf 
Molina, V. (Ed.). (2015). Panorama de los centros y programas de escritura en Latinoamérica. Cali: Pontificia Universidad Javeriana de Cali.

Moore, R. (1950). The writing clinic and the writing laboratory. National Council of Teachers of English, 11(7), 388-393.

North, S. (1984). The idea of a writing center. College English, 46(5), 433-446.

North, S. (2008). Revisiting “The idea of a Writing Center”. En R. Barnett y J. Blumner, The Longman Guide to Writing Center Theory and Practice (pp. 79-91). Nueva York: Pearson Education.

Núñez, J. (2013). Una aproximación a los centros de escritura en Iberoamérica. Legenda, 17(17), 64-102.

Pemberton, M. (1995). Rethinking the WAC/writing center connection. The Writing Center Journal, 15(2), 116-133.

Roscoe, R., y Chi, M. (2007). Understanding tutor learning: Knowledge-building and knowledge-telling in peer tutor's explanations and questions. Review of Educational Research, 77(4), 534-574. DoI: https:// doi.org/10.3102/0034654307309920.

Roscoe, R., y Chi, M. (2008). Tutor learning: The role of explaining and responding to questions. Instructional Science, 36(4), 321-350.

Rosenshine, B., Meister, C., y Chapman, S. (1996). Teaching students to generate questions: A review of the intervention studies. Review of Educational Research, 66(2), 181-221.

Sadker, M., y Sadker, D. (1993). Técnicas para la elaboración de preguntas. En J. Cooper (Coord.), Estrategias de enseñanza (pp. 173-228). México: Limusa.

Shamoon, L., y Burns, D. (1995). A critique of pure tutoring. The Writing Center Journal, 15(2), 135-151.

Summerfield, J. (2008). Writing centers: A long view. En R. Barnett y J. Blumner, The Longman Guide to Writing
Center Theory and Practice (pp. 22-28). Nueva York: Pearson Education.

Tan, B. (2011). Innovating writing centers and online writing labs outside North America. Asian EFL Journal, 13(2), 391-418.

Tzu-Shan, C. (2013). The idea of a writing center in asian countries: A preliminary search of models in Taiwan. Praxis: A Writing Center Journal, 10(2), 1-9.

Vail, A., Grafsgaard, J., Boyer, K., Wiebe, E., y Lester, J. (2016). Predicting learning from student affective response to tutor questions. En A. Micarelli, J. Stamper y K. Panourgia (Eds.), Panourgia, Intelligent Tutoring Systems, $13^{\text {th }}$ International Conference, ITS (pp. 154-164). Zagreb, Croatia: Springer International Publishing.

Van Rensburg, W. (2006). Making academia transparent: Negotiating academic identities in the writing center. Recuperado de https://zeitschrift-schreiben.eu/ globalassets/zeitschrift-schreiben.eu/2006/rensburg_academicidenities.pdf.

Voigt, A., y Girgensohn, K. (2015). Peer tutoring in academic writing with non-native writers in a German writing center-Results of an empirical study. Journal of Academic Writing, 5(1), 65-73.

Vygotsky, L. (2001). Psicología pedagógica: un curso breve. Buenos Aires: Aique.

Waldo, M. (1993). The last best place for writing across the curriculum: The writing center. WPA: Writing Program Administration, 16(3), 15-26.

Waller, S. (2002). A Brief History of University Writing Centers: Variety and Diversity. Recuperado de http://www. newfoundations.com/History/WritingCtr.html

Widdowson, H. (1978). Teaching Language as Communication. Hong Kong: Oxford University Press.

Zuleta Araújo, O. (2005). La pedagogía de la pregunta, una contribución para el aprendizaje. Educere, 9(28), 115-119.

How to reference this article: Calle-Arango, L. (2019). La pregunta en las tutorías de los centros de escritura. Íkala, Revista de Lenguaje y Cultura, 24(1), 137-152. DoI: 10.17533/udea.ikala.v24n01 a07 